\title{
CT, MR and SPECT imaging in temporal lobe epilepsy
}

Roderick Duncan, James Patterson, Donald M Hadley, Peter Macpherson, Martin J Brodie, Ian Bone, Anne P McGeorge, David J Wyper

\begin{abstract}
Cranial computed tomography (CT) with modified temporal lobe technique, $0 \cdot 15 \mathrm{~T}$ magnetic resonance imaging (MRI) and single photon emission computed tomography (SPECT) were carried out on 30 patients with intractable temporal lobe epilepsy. Lateralising abnormalities were detected in 21/30 patients overall. Specific lesions were detected by CT in one patient and by MRI in seven patients (in one case bilateral). In addition CT detected asymmetry of the sylvian fissures or temporal horns in 10 patients, and MRI in eight patients. SPECT detected lateralising abnormalities in 19 patients (in five cases bilateral). It is concluded that low field MRI is superior to modified CT in demonstrating subtle structural lesions of the temporal lobe. Functional scanning with SPECT supports the evidence of origin of an epileptic focus in a substantial proportion of cases and may improve the selection of patients for surgery.
\end{abstract}

Temporal lobe epilepsy is the commonest form of epilepsy encountered and is often refractory to drug therapy; the prevalence rate of such refractory epilepsy may be as high as 80-90/ 100,000 in the general population. ${ }^{1}$ Temporal lobectomy is increasingly recognised as an effective treatment, giving good results in 70$80 \%$ of patients, with low morbidity and mortality. ${ }^{23}$ Its use has been restricted to a great extent by the complex and time consuming electrophysiological testing often required to lateralise the focus. New imaging techniques, however, may detect lateralising abnormalities, improving selection for surgery.

Institute of
Neurological Sciences,
Southern General
Hospital, Glasgow
R Duncan
J Patterson,
D M Hadley
P Macpherson
M J Brodie
I Bone
A P McGeorge
D J Wyper
Correspondence to:
Dr R Duncan,
Institute of Neurological
Sciences,
Southern General Hospital,
Govan Road,

Govan Road,

Glasgow G51 4TF, Scotland

Received 10 March 1989 and in revised form 6 July 1989. Accepted 19 July 1989. \begin{abstract}
ness), and features of temporal lobe involvement in the aura, such as deja vu, or in

Subjects

female) seen sequentially at two outpatien ranged from 13-47 (mean 27.9) years. The (mean 14.8 ) seizure disorder ranged from temporal lobe epilepsy and intractability. The former required both the absence (stare combined with impaired awareness and responsive-
\end{abstract}

the seizure itself, such as automatism. The latter required that seizure control be unsatisfactory despite optimal drug treatment. "Unsatisfactory" seizure frequency in this study ranged from one per fortnight to five per day. This depended on the occupation, social circumstances and expectations of the patient, the manifestations of the seizures themselves (brief absences being, for example, better tolerated than prolonged automatisms), and the duration of post ictal effects. The patients had all been tried on three major anticonvulsant drugs, separately or in combination, all with concentrations within their respective target ranges.

\section{Scanning procedure}

The patients were assessed clinically and scanned in hospital as day cases.

\section{HM-PAO SPECT}

HM-PAO is a lipophilic amine. It crosses the blood-brain barrier freely, and $85 \%$ of brain uptake occurs on the first pass after an intravenous injection. Once inside the brain tissue, it forms a hydrophilic compound. This can only slowly recross the blood-brain barrier and so its pattern of distribution in the brain, and that of the technetium $99 \mathrm{~m}$ with which it is labelled, reflects the amount delivered and hence $\mathrm{rCBF}$ at the time of the first pass after intravenous injection. The activity in brain tissue remains essentially constant for several hours, ${ }^{4}$ allowing a delay before scanning. Since rCBF correlates closely with regional cerebral metabolism in epilepsy, ${ }^{5}$ HM-PAO SPECT effectively images both

The patients were injected intravenously with $500 \mathrm{MBq}$ of $\mathrm{Tc} 99 \mathrm{~m}$ labelled HM-PAO during EEG recording using the standard 10/ 20 system of electrode placement. The time since the last seizure was recorded at the time of injection. All were scanned within one hour of injection using a Novo 810 tomographic imager, producing $12 \mathrm{~mm}$ axial sections parallel to the orbito-meatal line. The resolution of this system is less than $1 \mathrm{~cm}$, and the images are comparable to those of most positron emission tomography (PET) systems currently in use.

The SPECT images were interpreted independently by a clinician (RD), a radiologist (DMH) and a physicist (JP), blind to any data other than the diagnosis of temporal lobe epilepsy. Differences were resolved by consensus conference. The perfusion of each temporal lobe was compared not only with the contralateral temporal lobe, but also with the 
Table Summary of results of CT, MRI and SPECT

\begin{tabular}{lrrr}
\hline & $C T$ & MRI & SPECT \\
\hline Normal & 19 & 17 & 11 \\
Lateralising lesion shown & 1 & 7 & 19 \\
Asymmetry of sylvian fissure or temporal horn shown & 10 & 8 & $\frac{1}{5}$ \\
Bilateral abnormalities shown & 0 & 1 & 5 \\
\hline
\end{tabular}

Figure 1 Venn diagram showing the numbers of patients showing specific abnormalities on $C T$, $M R I$ and SPECT. The areas of intersection between circles represent patients with lesions showing on more than one imaging modality.

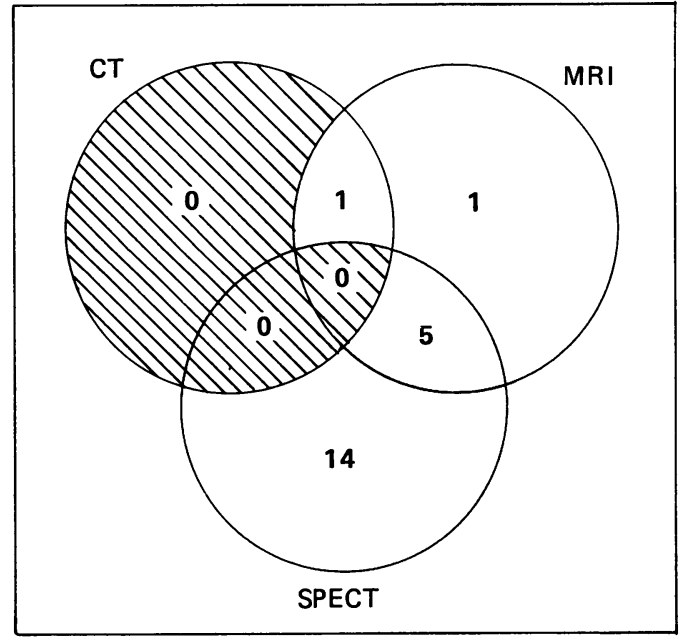

frontal and occipital lobes, allowing the distinction to be made between hypoperfusion of one temporal lobe and hyperperfusion of the other. Based on data from 10 normal volunteers, our upper limit of normal for side-to-side difference is $7 \%$. This is in agreement with data from other centres using the same technique. ${ }^{6}$

\section{$C T$ and $M R I$}

CT was carried out using a Phillips 350 Tomoscan, producing axial $6 \mathrm{~mm}$ sections of the whole brain, together with axial $6 \mathrm{~mm}$ temporal lobe sections angled parallel to the temporal horns, the latter cuts repeated after $50 \mathrm{ml}(300 \mathrm{mg} / \mathrm{ml}$ iodine $)$ of intravenous contrast medium.

MRI was performed using a Picker $0 \cdot 15 \mathrm{~T}$ resistive imager, producing $8 \mathrm{~mm}$ axial
SE 2000/80 T2 weighted images of the whole brain, axial $8 \mathrm{~mm}$ IR T1 weighted 1660/400/40 images of the temporal lobes, and coronal $5 \mathrm{~mm}$ balanced SE 700/32 images of the temporal lobes.

$C T$ and $M R$ images were interpreted and reported independently by two investigators (DMH and PM), blind to any data other than the diagnosis of temporal lobe epilepsy. Disagreements between reports were resolved by consensus conference.

\section{Results}

The results of CT, MRI and SPECT are summarised in table 1 . Lateralising abnormalities were seen in $21 / 30$ patients overall, in $15 / 21$ unilateral and in $6 / 21$ bilateral. The correspondence between the imaging modalities is illustrated by a Venn diagram in fig 1 .

\section{$C T$}

CT detected a specific lateralising abnormality in only one patient, a small area of calcification in the temporal lobe (fig 2). In a further 10 cases varying degrees of asymmetry of the temporal horns or sylvian fissures was apparent.

\section{$M R I$}

MRI detected specific lateralising abnormalities in seven patients. Areas of abnormal signal compatible with oedema, gliosis or tumour were detected in the medial temporal lobe in two patients (fig 3 ) and in the temporal pole in one patient. In one further patient a similar lesion in the medial temporal lobe was accompanied by an increase in signal throughout the contralateral temporal lobe. In two patients an increase in signal throughout one temporal lobe was seen as an isolated finding. In one patient a lesion compatible with an arteriovenous malformation or haemartoma was seen in the medial temporal lobe. In eight patients varying degrees of asymmetry of the temporal horns or sylvian fissures were seen.
Figure 2 A 23 year old man with frequent complex partial seizures. (a) CT showing punctate focus of calcification in the white matter of the right temporal lobe. (b) $T 2$ weighted (SE 2000/80) $M R I$ section showing the same lesion as a hypointense focus surrounded by an area of hyperintensity.
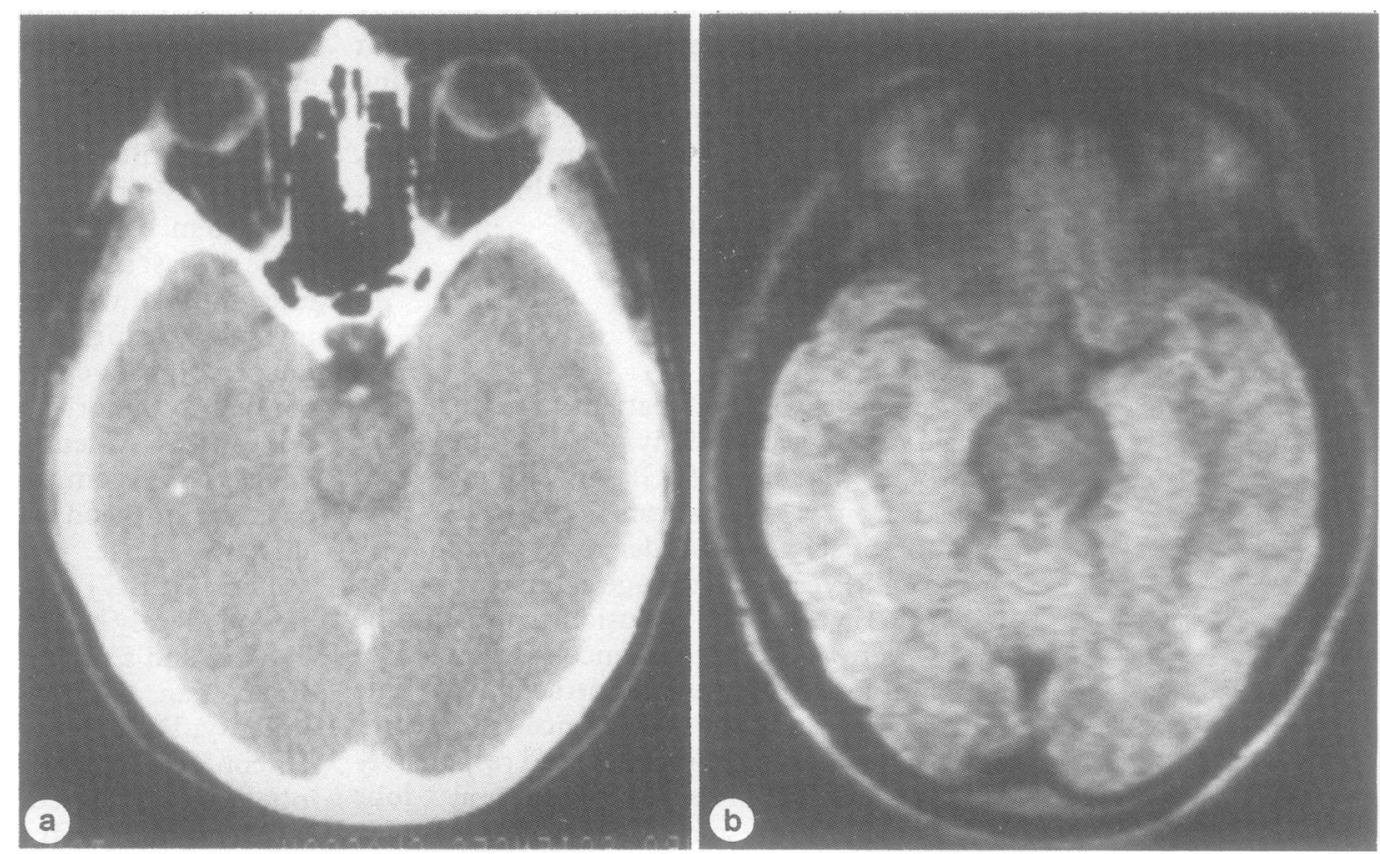
Figure 3 A 29 year old woman with severe complex partial and secondary generalised seizures. (a) SPECT 3 hours following a seizure showing focal hyperperfusion in the medial left temporal cortex (arrow). (b) T2 weighted (SE 2000/80) MRI section showing the hyperperfused lesion as an area of hyperintensity which is slightly space occupying. Anatomical asymmetry between temporal lobes is also shown.
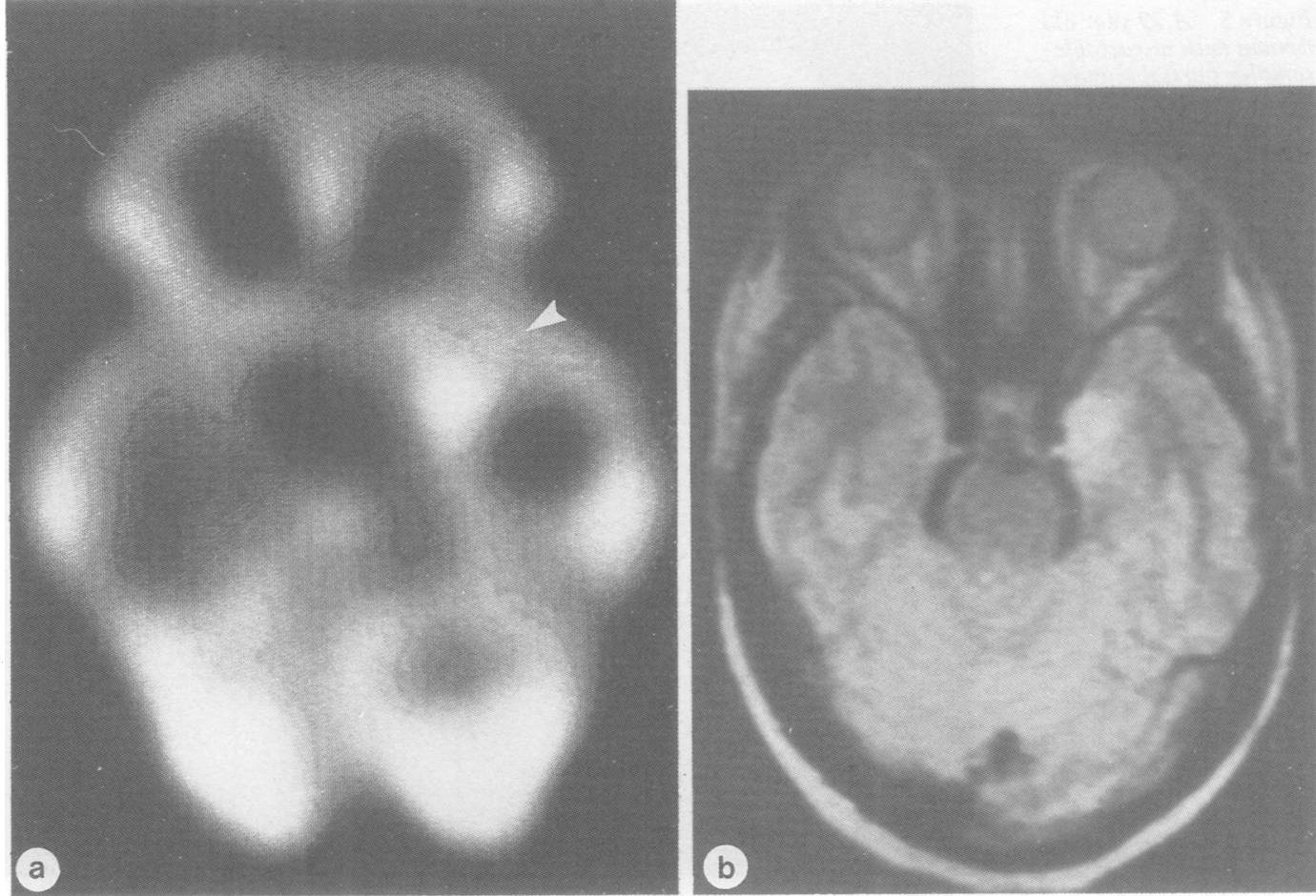

Comparison of $C T$ and $M R I$

MRI failed to detect calcification in one lesion detected by CT, but did detect the lesion as an area of high signal with a central area of low signal. Otherwise MRI detected lesions in six patients which were missed by $\mathrm{CT}$.

\section{SPECT}

SPECT detected abnormalities in 19 patients. In 10 this consisted of unilateral hypoperfusion confined to the temporal lobe (fig 4). In one of these patients an immediately post ictal scan showed an area of hyperperfusion at the same site.

In two patients temporal hypoperfusion extended into the frontal lobe. In one patient

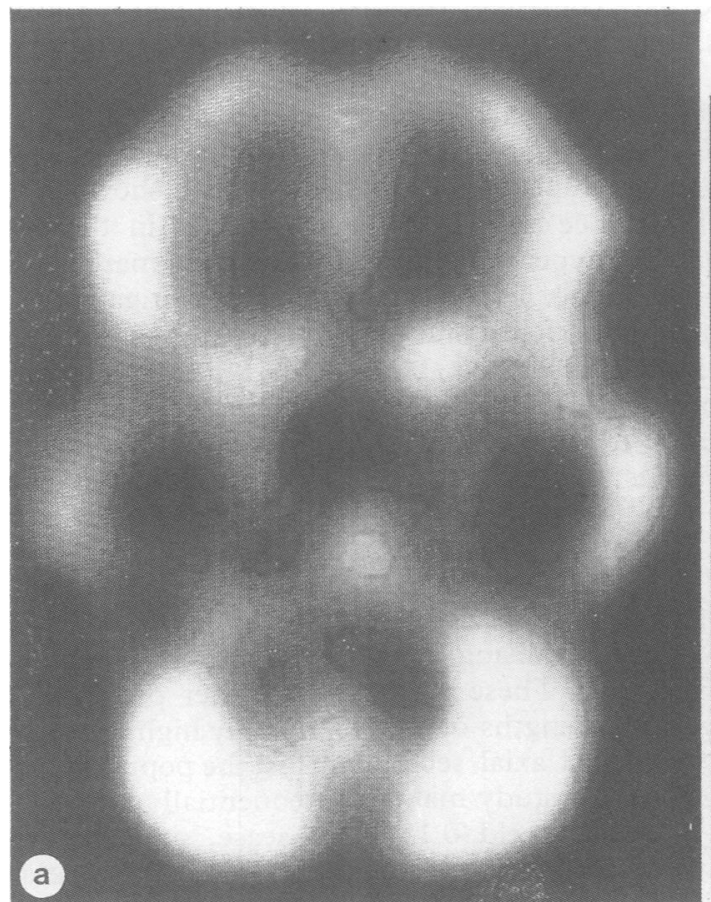

there was unilateral frontal hypoperfusion, in one patient bilateral frontal hypoperfusion and in one patient bilateral temporal hypoperfusion. In three patients temporal hypoperfusion was accompanied by contralateral temporal hyperperfusion. In one patient who had a seizure several minutes before injection of HM-PAO, SPECT showed temporal hyperfusion extending into the frontal lobe (fig 5 ).

\section{Comparison of SPECT with CT and MRI}

(comparison of "functional" with "structural" scans)

SPECT detected abnormalities in 14 patients in whom CT and MRI were normal, or showed only anatomical asymmetry. In seven of these

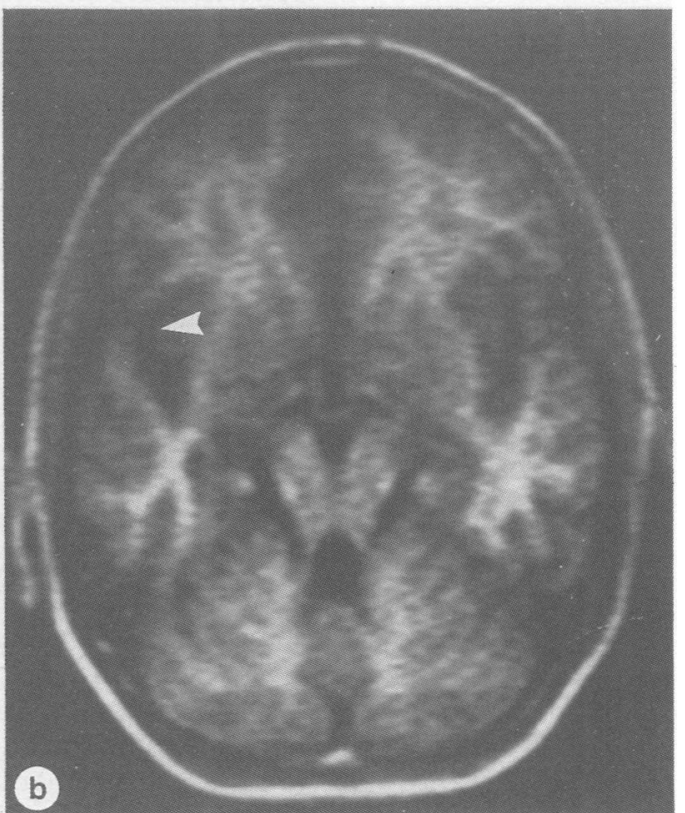

Figure 4 A 13 year old boy with intractable complex partial and secondary generalised seizures. (a) Interictal SPECT showing

hypoperfusion of the right temporal lobe. (b) $T 1$ weighted (IR 1400/400) 40) MRI section showing minimal enlargement of the right sylvian fissure (arrow). 
Figure 5 A 29 year old woman with intractable complex partial seizures. (a) SPECT 5 hours following a seizure showing marked asymmetry representing hyperperfusion of the left temporal and frontal cortex. (b) Balanced (SE 700/32) coronal MRI section through the frontal and mid temporal lobes showing no anatomical abnormality.
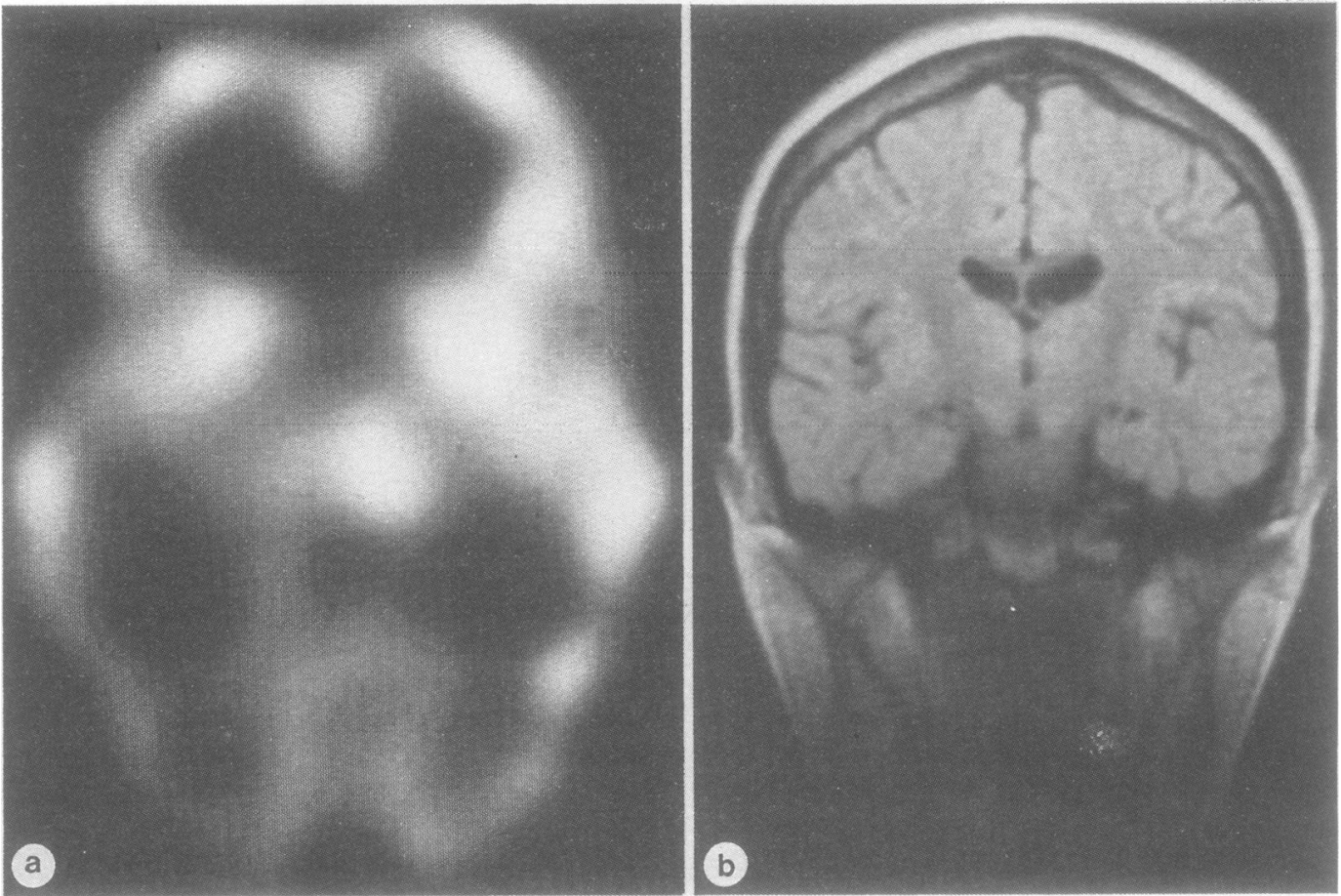

patients the abormalities were unilateral. In two cases lateralising abnormalities were seen on CT or MRI which were not accompanied by abnormalities of regional cerebral perfusion.

There were no cases where CT or MRI showed a definite lesion on one side and SPECT lateralised to the other, although there was one patient who showed CT and MRI enlargement of one temporal horn, but SPECT showed hypoperfusion of the contralateral temporal lobe. EEG lateralisation agreed with SPECT in this case.

\section{Correlation of CT, MRI with SPECT with surface EEG}

Multiple surface EEG recordings (including sleep or activated recordings in three cases, ictal recordings in three cases and sphenoidal recordings in one case) gave consistent lateralisation in 17 patients. There were lateralising abnormalities on CT, MRI or SPECT in 10 of these patients. Lateralisation agreed in all cases but one, where EEG lateralisation disagreed with lateralisation based on temporal hypoperfusion seen on SPECT. In three cases, SPECT showed hypoperfusion with hyperperfusion in the contralateral temporal lobe. In all three, EEG lateralised to the hypoperfused side and in two of these cases MRI also showed abnormalities on the hypoperfused side. In both the latter the hypoperfused temporal lobe was removed, with good results.

\section{Discussion}

Several reports have shown the superiority of MRI over CT in detecting lateralising lesions in temporal lobe epilepsy. ${ }^{7-12}$ These studies compared MRI using field strengths of 0.35 $1.5 \mathrm{~T}$, and CT using standard axial sections with or without contrast. This study makes a different comparison; that of low field $(0 \cdot 15 \mathrm{~T})$ MRI (most commonly available in the United Kingdom) and of CT with temporal lobe cuts angled to image temporal lobe structures to the best effect. Nonetheless, the difference between the results obtained using the two imaging modalities was the same; MRI is more sensitive in showing subtle lesions of temporal lobe structures, with the minor exception of small areas of calcification, which are better shown by CT. It is difficult to make direct comparisons in terms of the abnormality rate, rate of $20-25 \%$ in patients with normal $\mathrm{CT}^{7-10}$ whatever the field strength of the MR imager, with the interesting exception of one study comprising only well controlled patients which showed an abnormality rate of just over $50 \%{ }^{11}$ One retrospective study of patients who had had temporal lobectomy found abnormalities in $65 \%$ of patients with normal CT, but some patients had CT using low resolution equipment. ${ }^{12}$ Only one study found any lesion detected by CT and not by MRI, ${ }^{7}$ a small fleck of calcification.

In this study CT was marginally more effective in showing anatomical asymmetry. However, in those patients in whom asymmetry was marked (six cases), it was shown by both investigations. In those in whom asymmetry was shown by only one investigation (by MRI in two cases and by CT in four cases), the degree was minor and of little value as a lateralising finding.

Interictal studies in temporal lobe epilepsy using both positron emission tomography (PET) and SPECT have found focal temporal hypoperfusion as the most common abnormality. ${ }^{13} 14$ While this was the case in our own study, the proportion of patients (8/19) showing other patterns of $\mathrm{rCBF}$ abnormality was notably high. There was no obvious reason for this; the population in the present study was of sequentially selected patients with intractable disease, similar to those of other studies, ${ }^{15-17}$ and there is no reason to suppose that HMPAO SPECT is more likely to detect such but most studies show an MRI abnormality 
abnormalities. ${ }^{4}$ Their detection is important, as bilateral abnormalities of rCBF must be regarded as representing at least a relative contraindication to surgery. A possible exception to this is where an interictal scan shows hyperperfusion contralateral to temporal hypoperfusion. Three such cases appear in this series, and EEG, CT and MRI supported lateralisation to the hypoperfused side. Two of these patients have been operated on and remain seizure free at nine months follow up.

Past studies have found that rCBF studies (PET or SPECT) detect lateralising abnormalities in a higher proportion of patients than either CT or MRI, ${ }^{510^{15-18}}$ as was the case in our study.

The correlation between lateralisation based on single surface EEG recording and that based on hypoperfusion seen on PET or SPECT is poor, but improves as the results of multiple recordings are taken into account and becomes very good when the results of all EEG investigations, including depth electrodes etc, are taken into account ${ }^{18}$ Although the EEG investigations of our patients were largely based on multiple surface recordings, the correlation with SPECT was excellent with only one disagreement. In the one case where CT and MRI showed enlargement of one sylvian fissure and EEG lateralised to the other side, SPECT lateralisation agreed with EEG. There were otherwise no disagreements between scan results and EEG. Complex partial seizures with evidence of temporal lobe involvement may originate in extratemporal areas. Among the many interictal PET and SPECT studies in the literature there appear no patients with hypoperfusion confined to one temporal lobe who have seizures of extratemporal origin. Such PET and SPECT findings should therefore be regarded as at least supportive of a temporal origin for seizures. Localisation within the temporal lobe is not currently possible using interictal PET or SPECT.

The results of this study show that low field MRI is superior to modified CT in detecting abnormalities of the temporal lobe and suggest that SPECT should become part of the standard workup for temporal lobectomy. The latter investigation may prove a particularly powerful lateralising tool when used to image the focal increases in blood flow seen at the site of a focus during or just after a seizure. ${ }^{19}{ }^{20} \mathrm{EEG}$ is likely to remain the main lateralising investigation in most patients, but the use of techniques such as MRI and SPECT may not only increase the number of patients suitable for surgery but, by improving patient selection, may improve outcome in terms of the propor- tion of patients who benefit. In Glasgow we are embarking on a programme of temporal lobectomy with continuous audit of investigations and results. We hope that this will identify those techniques, whether imaging or electrophysiological, which are most effective in achieving a high success rate.

The SPECT scanner is funded by a grant from the Wellcome Trust to Professor J McCulloch, Wellcome Surgical Institute, University of Glasgow and Professor M Brooks, Department of Psychological Medicine, University of Glasgow.

1 Keranen T, Reikkinen P. Severe epilepsy: diagnostic and epidemiological aspects. Acta Neurol Scand 1988;78:7-14.

2 Olivier A. Risk and benefit in the surgery of epilepsy: complications and positive results on seizure tendency and complications and positive results on seizure tendency and
intellectual function. Acta Neurol $S$ cand 1988;78: intellect 21 .

3 Cahan LD, Engel J. Surgery for epilepsy: a review. Acto Neurol Scand 1986;73:551-60.

4 Neirinckx RD, Canning LR, Piper IM, et al. Technetium 99m d,1-HM-PO: a new radiopharmaceutical for SPECT imaging of regional cerebral blood perfusion. $J$ Nucl Med 1987;28:191-202.

5 Kuhl DE, Engel J, Phelps ME, Selin C. Epileptic patterns of local cerebral metabolism and perfusion in humans determined by emission computed tomography of $18 \mathrm{FDG}$ and 13NH3. Ann Neurol 1980;8:348-60.

6 Podreka I, Lang W, Suess E, et al. Hexamethylpropyleneamine oxime (HM-PAO) single photon emission computed tomography (SPECT) in epilepsy. Brain Tomoputed tomography

7 Schorner W, Meenke HJ, Felix R. Temporal lobe epilepsy: comparison of CT and MR imaging. Am J Neuroradio 1987;8:773-81

8 Lesser RF, Modic MT, Weinstein MA, et al. Magnetic resonance imaging $(1.5 \mathrm{Tesla})$ in patients with intractable focal seizures. Arch Neurol 1986;43:367-71.

9 Sperling MR, Wilson G, Engel J, Babb TL, Phelps M, Bradley $M$. Magnetic resonance imaging in intractable partial epilepsy: correlative studies. Ann Neurol 1986;20:57-62.

10 Theodore WH, Dorwart R, Holmes M, Porter RJ, DiChiro $G$. Neuroimaging in refractory partial seizures. CompariG. Neuroimaging in refractory partial seizures. Compari
son of PET, CT and MRI. Neurology 1986;36:750-9.

11 Triulzi F, Francheschi M, Fazzio F, Del Maschio A. Nonrefractory temporal lobe epilepsy: 1.5 T MR imaging. Radiology 1988;166:181-5.

12 Kuzniecky R, DeLaSayette D, Ethier R, et al. Magnetic resonance imaging in temporal lobe epilepsy: pathological correlations. Ann Neurol 1987;22:341-7.

13 Hougaard K, Oikawa T, Sveinsodittir E, Skinhoj E, Ingva $\mathrm{DH}$, Lassen NA. Regional cerebral blood flow in focal cortical epilepsy. Arch Neurol 1976;33:527-35.

14 Engel J. The use of positron emission tomographic scanning in epilepsy. Ann Neurol 1984;15:S180-191.

15 Kuhl DE, Fishbein D, Dubinsky R. Patterns of cerebral metabolism in patients with partial seizures. Ann Neurol 1988;38:1201-6.

16 Abou Khalil BW, Siegel JG, Sackellares C, Gilman S, Hichwa R, MarshallR. Positron emission tomography studies of cerebral glucose metabolis
epilepsy. Ann Neurol 1987;22:480-6.

17 Theodore WH, Newmark ME, Sato S, Brooks R, Patronas N, DeLaPaz R. 18F Fluorodeoxyglucose positron emission tomography in refractory complex partial seizures. Ann Neurol 1983;14:429-37.

188 Engel J, Kuhl DE, Phelps ME, Crandall PH. Comparative localisation of epileptic foci in partial epilepsy by PCT and EEG. Ann Neurol 1982;12:529-37.

19 Lee BI, Markand ON, Wellman HN, et al. HIDPMSPECT in patients with medically intractable complex partial seizures. Arch Neurol 1985;45:397-402.

20 Rowe CC, Berkovic SS, Austin M, McKay WJ, Bladin PF. Postictal STET in epilepsy. Lancet 1984;i:389-90. 